\title{
The rule of law and the effectiveness of civil justice
}

Citation for published version (APA):

Auersperger Matić, A. (2017). The rule of law and the effectiveness of civil justice: a critical reading of European human rights jurisprudence. [Doctoral Thesis, Maastricht University]. Maastricht University. https://doi.org/10.26481/dis.20170426aam

Document status and date:

Published: 01/01/2017

DOI:

10.26481/dis.20170426aam

Document Version:

Publisher's PDF, also known as Version of record

\section{Please check the document version of this publication:}

- A submitted manuscript is the version of the article upon submission and before peer-review. There can be important differences between the submitted version and the official published version of record.

People interested in the research are advised to contact the author for the final version of the publication, or visit the DOI to the publisher's website.

- The final author version and the galley proof are versions of the publication after peer review.

- The final published version features the final layout of the paper including the volume, issue and page numbers.

Link to publication

\footnotetext{
General rights rights.

- You may freely distribute the URL identifying the publication in the public portal. please follow below link for the End User Agreement:

www.umlib.nl/taverne-license

Take down policy

If you believe that this document breaches copyright please contact us at:

repository@maastrichtuniversity.nl

providing details and we will investigate your claim.
}

Copyright and moral rights for the publications made accessible in the public portal are retained by the authors and/or other copyright owners and it is a condition of accessing publications that users recognise and abide by the legal requirements associated with these

- Users may download and print one copy of any publication from the public portal for the purpose of private study or research.

- You may not further distribute the material or use it for any profit-making activity or commercial gain

If the publication is distributed under the terms of Article $25 \mathrm{fa}$ of the Dutch Copyright Act, indicated by the "Taverne" license above, 


\section{ENGLISH SUMMARY}

The present study examines, in the European context, the intricate relationship between the functioning of civil justice and the contemporary doctrine of human rights, which among other things includes the guarantee of a fair trial. The starting premise of the inquiry is that the issue of effective justice can be understood in the wider social context of increased expectations about the working of the legal system, reflected in the contemporary global discourse on the rule of law. This ideal is based on a series of related considerations, thoughts and values about the place law ought to hold in society and the minimal conditions for its legitimacy. Unlike its original versions that mainly underlined constitutional protection of rights of individuals, the current rule of law discourse - which might be termed 'institutional' - also emphasises functioning legal and judicial institutions as an important element. Most importantly, it emphasises the effectiveness of law as the crucial evaluative standard by which legal systems can be assessed.

Greater scrutiny of the role of judicial institutions in the civil law field has also become merited on account of the growth of international legal standards and their place in contemporary policymaking. This development is best seen in Europe, where legal integration now affects areas that had for a long time been considered to be a prerogative of national governments and legal communities. At the more general level, the Council of Europe has taken important steps forward in the formulation of common standards and expertise, including notably with the establishment of the European Commission for the Efficiency of Civil Justice (CEPEJ). Within the European Union, the creation of the common market and the free movement of persons have also quickened the pace of cooperation in the sphere of civil justice, as has the fact that it is national courts that actually enforce harmonised substantive rules in areas ranging from competition law to consumer protection. Moreover, the legally binding nature of the Charter of Fundamental Rights has given a new life to the drive to establish stricter standards on civil justice across Europe. This is why a number of European institutions, including the European Commission, now actively monitor the work of national judiciaries and undertake analytical reviews.

In formal terms, the most important standard that allows for legal scrutiny of the performance of national civil justice systems in Europe today is undoubtedly Article 6 of the European Convention on Human Rights on the right to a fair trial, as interpreted by the European Court of Human Rights. Over the last two decades in particular, the case law developed under this article has become very extensive and now covers many aspects of civil procedure. Significantly, in addition to placing 
minimal requirements concerning the fairness of proceedings, the Court has also created certain standards about the minimal criteria of institutional competence that must be guaranteed in European countries. The weight of this case law is now even greater due to the reception of this case law in the primary law of the European Union, which means that further obligations to respect these criteria have been undertaken by both Union institutions and Member States, within the framework of legal structures created by European Union treaties.

To evaluate the doctrines established by the Strasbourg Court, the study proposes a key operational concept - the effectiveness of civil justice - as a guiding standard that can be applied as a criterion of evaluation of the performance of civil justice institutions. Taking into account the various evaluation reports concerning civil justice systems in various countries as well as the insights of law and economics, it has been submitted that three criteria or parameters can form a common baseline of effectiveness, namely the length, cost and predictability of proceedings. These parameters serve not only as a list of thresholds, but also as a map of possible mutual interactions in terms of policy choices. The concept of effectiveness thus implicitly involves a corresponding policy model requiring reasonable and proportionate emphasis on the three parameters as well as balancing with the countervailing imperatives of fairness. When effectiveness is phrased as a legal argument, especially in the course of human rights adjudication, an analysis of interpretive practice must accordingly account for these three parameters and ensure coherence and balance in policy choices involved in the determination of respective thresholds.

The first parameter, length, is the only one based on explicit Convention wording of Article 6, guaranteeing a right to proceedings within a 'reasonable time'. This requirement has for a long time been one of the main areas of activity for the Strasbourg Court, indicating not only that court delays are a pressing issue in many civil justice systems in Europe, but also that attempts to find adequate solutions at the European level have not really been very successful. The Court has allowed states significant leeway in the organisation of their judicial systems and has, in particular, not commented on possible underlying causes of delay. It has also been rather timid when it comes to dealing with the consequences of frequent violations of the reasonable time requirement in legal systems where judicial delay is pervasive. The articulation of the issue of delay in terms of a right of individual litigants separate from the broader question of justice policy - either in terms of the procedural structure of the justice system or in terms of budgetary support appears to have obscured the complexity and systemic nature of the underlying problems. It has also diverted attention from overdue systemic reform to the establishment of further individual remedies, a solution that is however viable only if delay occurs in isolated cases. 
The second parameter, cost, has also given rise to an important body of case law. The Strasbourg Court has early on recognised in principle that in a limited set of circumstances, the principle of fair trial requires public funding of judicial remedies for economically weak claimants while at the same time recognising that reducing complexity of proceedings could also provide a possible solution. Later, the Court gradually started to develop its case law into a set of requirements regarding legal aid, while a series of related cases circumscribed the right of states to require the payment of ex ante court fees. On the issue of legal aid, the Court gradually became more specific, even to the point of prescribing particular forms and rules for the provision of assistance to persons in need for some legal systems, which does not however fit with the reserved approach adopted earlier. On the other hand, the issue of court fees has been addressed in a rather casuistic fashion, with violations found mainly in a few cases where extraordinarily high court fees had been imposed, but without a more comprehensive setting of a generally applicable standard. The overall result of this approach is that the treatment of cost from the angle of human rights law is somewhat uneven or incoherent, and the limited and circumstantial findings of violations stand in a contrast with the extensive and ambitious treatment of delay.

The third parameter, predictability, was dealt with in a different fashion. While the Strasbourg Court has acknowledged the importance of case law as a means of providing certain foreseeability in adjudication, it did not examine the important differences in appellate structures and case law practices between European jurisdictions which are however crucial for understanding the institutional background of the issue. Faced with complaints about the unequal treatment of similar situation by national courts, the Court initially refused to take any decisive position on the subject. Accordingly, decisional divergence, even to the point of obviously unequal treatment of identical situations, was seen - especially with reference to continental jurisdictions - as a side effect of having hierarchical and regionalised judicial systems with many instances and judicial panels within courts. It was only with the reception of cases from Eastern Europe that the Court realised how such structures - notably when coupled with a highly formalist legal practice that does not regard past cases as guidelines for decisions - can lead to absurd results, and it began to criticise national judgments as inconsistent. The operative concept used was 'legal certainty', and after a number of cases it appeared that the Court would be willing to impose the requirement to observe case law on European legal systems in general. Nevertheless, after several ad hoc attempts, the Court ultimately decided to take a step back, adopting an approach that requires consistency only conditionally.

The examination of European human rights law has accordingly shown that it now contains a number of identifiable principles regarding the effectiveness of civil justice, even if this term is not used in their articulation. The Court has 
identified the issues of length, cost and predictability as important from the viewpoint of human rights, and has attempted to lay down specific requirements in those fields with a lot of attention to the particularities of individual cases and the characteristics of the legal systems in question. Nevertheless, on close inspection the case law can be revealed to be problematic from a number of angles. Doctrines in the area of effectiveness of justice are fragmented and based on a large number of ad hoc decisions made without extensive systemic analysis, having as the consequence that the issues of length, cost and predictability are treated as separate problems rather as mutually related aspects of the more general problem of institutional design. The case law also appears to be incoherent in a number of ways. Firstly, the Court has applied different approaches in comparable situations, with different thresholds applied for different jurisdictions. Secondly, there are different degrees of emphasis for different issues, with the issue of length being subject to intensive scrutiny, pilot judgments and even an order of the Court to the respondent states to adopt special laws on national remedies, while the issue of cost and predictability are treated as too dependent on national institutional arrangements to warrant the imposition of more stringent European standards. Thirdly, there is also incoherence internally in the development of requirements in each of the three areas, with criteria ranging from very general and abstract at some points to very specific on others. In terms of outcomes, on the other hand, it transpires from Strasbourg jurisprudence that while the Court has professed to tackle seriously some of the most pressing issues of judicial effectiveness, the substance of requirements ultimately laid down is actually rather limited.

It is submitted that the uneven and for the most part modest result of these human rights doctrines can at least in part be explained by the decisional approach, which can be characterised as predominantly formalist or conceptualist. The Strasbourg Court has not used or referred to available national and international policy documents to analyse the problem of lengthy, costly and unpredictable proceedings, nor has it - with few exceptions - drawn inspiration from comparative law. Rather, it applied abstract imagination and abstract concepts like 'reasonable time', 'access to court' and 'legal certainty' to make its decisions, deriving abstract rights from the idea of a fair trial by asking the question whether a litigant has a 'right' to certain institutional arrangements in the legal system in question. The Strasbourg Court thus developed its doctrines without a frame of reference regarding evaluation criteria or policy choices that determine the institutional context in which civil justice functions. In particular, it has not delved into a more explicit balancing of fairness with effectiveness as guiding value orientations in the formation of respective procedural models, and has not examined the corresponding issues of resource allocation and incentives as possible problems underlying the difficulties faced by the justice systems under review. In other words, the approach of the Court has predominantly been based on the assumption that the 
issues of delay, cost and predictability - formulated as human rights - can be dissociated from important policy choices concerning the formation of civil justice institutions. In this sense, the doctrines developed by the Strasbourg Court can be said to be insufficiently attentive to the systemic nature of problems encountered by the legal systems under consideration, and the conceptualist decisional approach that it has applied can accordingly be criticised as leading to questionable results from the point of view of political legitimacy of judicial law-making in the European transnational legal framework. 


\section{RÉSUMÉ EN FRANÇAIS}

La présente étude examine, dans le contexte européen, la relation complexe entre le fonctionnement de la justice civile et la doctrine contemporaine des droits de l'homme, qui comprend entre autres la garantie d'un procès équitable. Le postulat de départ de l'étude est que la question de l'effectivité de la justice peut être comprise dans le contexte social plus large des attentes accrues du fonctionnement du système juridique, qui se reflète dans le discours mondial contemporain sur le principe de l'État de droit. Cet idéal est basé sur une série de considérations connexes, de pensées et de valeurs relatives à la place que la loi doit tenir dans la société et les conditions minimales pour sa légitimité. Contrairement à ses versions originales qui soulignaient principalement la protection constitutionnelle des droits des individus, le discours de l'État de droit actuel - qui pourrait être qualifié d' " institutionnel » - souligne également le fonctionnement des institutions judiciaires et juridiques comme un élément important. Plus important encore, il met l'accent sur l'effectivité de la loi comme la norme d'évaluation capitale par laquelle les systèmes juridiques peuvent être évalués.

Un examen plus approfondi du rôle des institutions de justice dans le domaine du droit civil revêt un intérêt d'autant plus important vu la croissance des normes juridiques internationales et de leur place dans l'élaboration des politiques contemporaines. Ce développement est le plus représentatif en Europe, où l'intégration juridique concerne désormais les domaines qui depuis longtemps avaient été considérés comme étant une prérogative des gouvernements nationaux et des communautés juridiques. Au niveau plus général, le Conseil de l'Europe a pris des mesures importantes en vue de l'élaboration de normes communes et d'expertise, y compris notamment avec la création de la Commission européenne pour l'efficacité de la justice (CEPEJ). Au sein de l'Union européenne, la création du marché commun et la libre circulation des personnes ont également accéléré le rythme de la coopération dans le domaine de la justice civile, tout comme le fait que ce sont les tribunaux nationaux qui font appliquer les règles de fond harmonisées dans des domaines allant du droit de la concurrence à la protection des consommateurs. En outre, le caractère juridiquement contraignant de la Charte des droits fondamentaux a donné une nouvelle vie en incitant à établir des normes plus strictes en matière de justice civile à travers l'Europe. C'est la raison pour laquelle un certain nombre d'institutions européennes, y compris la Commission européenne, désormais surveillent activement les travaux des instances judiciaires nationales et entreprennent des examens analytiques.

Sur le plan formel, la norme la plus importante qui permet un examen juridique des performances des systèmes nationaux de justice civile en Europe est sans aucun doute aujourd'hui l'article 6 de la Convention européenne des droits de 
l'homme sur le droit à un procès équitable, tel qu'interprété par la Cour européenne des droits de l'homme. Au cours des deux dernières décennies, en particulier, la jurisprudence, développée en vertu du présent article, est devenue très vaste et couvre maintenant de nombreux aspects de la procédure civile. En plus de placer les exigences minimales relatives à l'équité des procédures, la Cour a également dégagé, de manière significative, certaines normes sur les critères minimaux de compétence institutionnelle qui doivent être garantis dans les pays européens. Le poids de cette jurisprudence est maintenant encore plus grand en raison de l'intégration de cette jurisprudence dans le droit primaire de l'Union européenne, ce qui signifie que de nouvelles obligations de respect de ces critères ont été entreprises à la fois par les institutions de l'Union et par les États membres, dans le cadre des structures juridiques créées par les traités de l'Union européenne.

Pour évaluer les principes découlant de la jurisprudence de la Cour de Strasbourg, l'étude propose un concept opérationnel clé - l'effectivité de la justice civile - en tant que norme directrice qui peut être appliquée comme critère d'évaluation de la performance des institutions judiciaires. Compte tenu des divers rapports d'évaluation concernant les systèmes de justice civile dans les différents pays, ainsi que les idées du droit et de l'économie, il a été soutenu que trois critères ou paramètres peuvent former une base commune de l'efficacité, à savoir la durée, le coût et la prévisibilité des procédures. Ces paramètres servent non seulement de liste de seuils, mais aussi de carte des interactions mutuelles possibles en termes de choix politiques. Le concept d'effectivité implique donc implicitement un modèle de politique correspondant exigeant une attention raisonnable et proportionnée sur les trois paramètres ainsi que l'équilibre avec les impératifs compensatoires d'équité. Lorsque l'effectivité est formulée comme un argument juridique, en particulier dans le cadre de l'arbitrage des droits de l'homme, une analyse de la pratique interprétative doit donc tenir compte de ces trois paramètres et assurer la cohérence et l'équilibre dans les choix politiques impliqués dans la détermination des seuils respectifs.

Le premier paramètre, la durée, est le seul basé sur le libellé explicite de l'article 6 de la Convention, qui garantit le droit à un procès dans un 'délai raisonnable'. Cette exigence a depuis longtemps été l'un des principaux domaines d'activité de la Cour de Strasbourg, ce qui indique que non seulement l'arriéré judiciaire est un problème urgent dans de nombreux systèmes de justice civile en Europe, mais aussi que les tentatives de trouver des solutions adéquates au niveau européen n'ont pas vraiment été une réussite. La Cour a permis aux États une marge de manœuvre importante dans l'organisation de leurs systèmes judiciaires et en particulier, n'a pas fait de commentaires sur les causes sous-jacentes possibles de retard. Elle a également été plutôt timide quant à faire face aux conséquences des violations fréquentes de l'exigence du délai raisonnable dans les systèmes juridiques où le retard judiciaire est omniprésent. La formulation de la question du retard en 
termes de droit des plaideurs individuels séparée de la question plus large de la politique de la justice - soit en termes de la structure procédurale du système de justice soit en termes de soutien budgétaire - semble avoir occulté la complexité et le caractère systémique des problèmes sous-jacents. Cela a également détourné l'attention de la réforme systémique attendue vers la mise en place d'autres voies de recours individuels, une solution qui n'est cependant viable que si le retard se produit dans des cas isolés.

Le second paramètre, le coût, a également donné lieu à un important corpus de jurisprudence. La Cour de Strasbourg a dès le début reconnu en principe que dans un nombre limité de circonstances, le principe du procès équitable nécessite un financement public des recours judiciaires pour les demandeurs économiquement faibles, tout en reconnaissant en même temps que la réduction de la complexité de la procédure pourrait également fournir une solution possible. Plus tard, la Cour a progressivement commencé à développer sa jurisprudence dans un ensemble d'exigences en ce qui concerne l'aide juridique, tandis qu'une série de cas connexes a circonscrit le droit des États à exiger le paiement des frais de justice ex ante. Sur la question de l'aide juridique, la Cour devient progressivement plus spécifique, au point même de prescrire des formules et des règles particulières pour apporter une assistance aux personnes dans le besoin pour certains systèmes juridiques, qui cependant ne correspondent pas à l'approche réservée adoptée plus tôt. D'autre part, la question des frais de justice a été abordée d'une façon assez casuistique, avec des violations constatées principalement dans quelques cas où des frais de justice extraordinairement élevés avaient été imposés, mais sans un cadre plus complet d'une norme généralement applicable. Le résultat global de cette approche est que le traitement des coûts dans le cadre de la législation des droits de l'homme est quelque peu inégal ou incohérent, et les découvertes limitées et circonstancielles de violations reposent dans un contraste avec le traitement vaste et ambitieux du retard.

Le troisième paramètre, la prévisibilité, a été traité d'une façon différente. Alors que la Cour de Strasbourg a reconnu l'importance de la jurisprudence comme un moyen de fournir certaines prévisibilités dans l'arbitrage, elle n'a pas examiné les différences importantes dans les structures d'appel et les pratiques de la jurisprudence entre les juridictions européennes qui sont pourtant essentielles pour comprendre le contexte institutionnel de la question. Face à des plaintes concernant un traitement inégal de situation similaire par les juridictions nationales, la Cour a d'abord refusé de prendre une position décisive sur le sujet. Par conséquent, la divergence de décision, même au point d'un traitement manifestement inéquitable de situations identiques, a été observé - en particulier en référence aux juridictions continentales - comme un effet secondaire d'avoir des systèmes judiciaires hiérarchiques et régionalisés avec de nombreux instances et plusieurs chambres au sein des tribunaux. Ce fut seulement après avoir reçu des cas provenant d'Europe de 
l'Est que la Cour a réalisé combien ces structures - notamment lorsqu'elles sont associées à une pratique juridique très formaliste qui ne considère pas les cas passés comme lignes directrices dans les décisions - peuvent conduire à des résultats absurdes, qu'elle a commencé à critiquer les jugements nationaux comme incohérents. Le concept opératoire utilisé était la « sécurité juridique », et après un certain nombre de cas, il est apparu que la Cour serait disposée à imposer l'obligation de suivre la jurisprudence sur les systèmes juridiques européens en général. Néanmoins, après plusieurs tentatives ad hoc, la Cour a finalement décidé de prendre un peu de recul, en adoptant une approche qui exige de la cohérence sous certaines conditions.

L'examen de la législation européenne sur les droits de l'homme a donc montré qu'elle contient maintenant un certain nombre de principes identifiables quant à l'effectivité de la justice civile, même si ce terme n'est pas utilisé dans son discours. La Cour a identifié l'importance des problèmes de durée, de coût et de prévisibilité du point de vue des droits de l'homme, et a tenté de définir des exigences spécifiques dans ces domaines avec beaucoup d'attention aux particularités des cas individuels et des caractéristiques des systèmes juridiques en question. Néanmoins, l'examen minutieux de la jurisprudence peut révéler celle-ci problématique sous un certain nombre d'angles. La jurisprudence dans le domaine de l'effectivité de la justice est fragmentée et basée sur un grand nombre de décisions ad hoc prises sans analyse systémique étendue, ayant comme conséquence que les questions de durée, de coût et de prévisibilité sont traitées comme des problèmes séparés plutôt que des aspects mutuellement connexes du problème plus général de la conception institutionnelle. La jurisprudence semble également être incohérente dans un certain nombre de façons. Premièrement, la Cour a appliqué des approches différentes dans des situations comparables, avec différents seuils appliqués pour différentes juridictions. Deuxièmement, il existe différents degrés d'importance pour différents problèmes, avec la question de la durée étant soumise à un contrôle intensif, à des arrêts pilotes et même à un ordre de la Cour pour les États défendeurs d'adopter des lois spéciales sur les recours nationaux, alors que la question du coût et de la prévisibilité est traitée comme étant trop dépendante des dispositions institutionnelles nationales pour justifier l'imposition de normes européennes plus strictes. Troisièmement, il existe également une incohérence interne dans le développement des exigences dans chacun des trois domaines, avec des critères allant de très général et abstrait à certains points très précis pour d'autres. En termes de résultats, d'autre part, il ressort de la jurisprudence de Strasbourg que si la Cour a professé d'attaquer sérieusement quelques-uns des problèmes les plus urgents de l'effectivité judiciaire, la substance des exigences finalement établies est en fait assez limitée.

Il est soutenu que le résultat inégal et modeste en grande partie de ces principes des droits humains peut au moins en partie être expliqué par l'approche 
décisionnelle, qui peut être caractérisée comme essentiellement formaliste ou conceptualiste. La Cour de Strasbourg n'a pas utilisé ou mentionné des documents de politique nationaux et internationaux disponibles pour analyser le problème des procédures longues, coûteuses et imprévisibles, ni ne s'est - à quelques exceptions près - inspirée de droit comparé. Au contraire, elle a appliqué une imagination et des concepts abstraits comme un « délai raisonnable », l' « accès à la justice » et la « sécurité juridique » pour prendre ses décisions, découlant des droits abstraits de l'idée d'un procès équitable en posant la question de savoir si un plaideur a « droit » à certaines dispositions institutionnelles dans le système juridique en question. La Cour de Strasbourg a ainsi développé ses principes sans un cadre de référence en ce qui concerne les critères d'évaluation ou les choix politiques qui déterminent le contexte institutionnel dans lequel la justice civile fonctionne. En particulier, elle n'a pas puisé dans un équilibre plus explicite d'équité avec efficacité en tant qu'orientation à valeur directrice dans la formation de modèles procéduraux respectifs, et elle n'a pas non plus examiné les questions correspondantes de l'allocation des ressources et des incitations comme d'éventuels problèmes sousjacents aux difficultés rencontrées par les systèmes de justice en cours d'examen. En d'autres termes, l'approche de la Cour a surtout été fondée sur l'hypothèse que les questions de délai, de coût et de prévisibilité - formulées en tant que droits de l'homme - peuvent être dissociées des choix importants concernant la formation des institutions de justice. En ce sens, les principes développées par la Cour de Strasbourg peuvent être définies comme insuffisamment attentifs au caractère systémique des problèmes rencontrés par les systèmes juridiques à l'étude, et l'approche décisionnelle conceptualiste qu'elle a appliqué peut dès lors être critiquée comme conduisant à des résultats douteux du point de vue de la légitimité politique du droit de décision judiciaire dans le cadre juridique européen transnational. 


\section{NEDERLANDSE SAMENVATTING}

Deze studie onderzoekt in een Europese context de complexe relatie tussen de werking van het justitie en de hedendaagse leer op het gebied van mensenrechten, waaronder de garantie op een eerlijk proces. Het uitgangspunt bij dit onderzoek is dat vraagstukken omtrent effectieve gerechtigheid kunnen worden begrepen in de bredere sociale context waarin verwachtingen van het rechtssysteem toenemen, wat tot uiting komt in de hedendaagse wereldwijde discussie over de beginselen van rechtstaten. Dit ideaal stoelt op een reeks samenhangende overwegingen, gedachten en waardes over de rol die het recht zou moeten innemen in de maatschappij, en over de bijbehorende minimumvereisten voor haar legitimiteit. In tegenstelling tot eerdere discussies, waarin vooral aandacht werd besteed aan de grondwettelijke bescherming van de rechten van individuen, wordt in de huidige discussie over de beginselen van de rechtstaat - ook wel 'institutioneel' te noemen - ook het belang van werkende wetgevende en rechtsprekende instituties benadrukt. Hierbij wordt vooral ook nadruk gelegd op de effectiviteit van de rechtspraak als essentiële evaluatienorm voor het beoordelen van rechtssystemen.

Meer aandacht voor de rol van juridische instituties in het civiele recht is ook nodig vanwege de toename van internationale juridische standaarden, en hun rol in hedendaagse beleidsprocessen. Deze ontwikkeling is het beste te zien in Europa, waar juridische integratie inmiddels gevolgen heeft voor terreinen die lange tijd beschouwd werden als toebehorend aan nationale overheden en juridische gemeenschappen. Meer in het algemeen heeft de Raad van Europa belangrijke stappen voorwaarts gezet middels het opstellen van gemeenschappelijke normen en expertises, in het bijzonder door het oprichten van de Europese Commissie voor de Efficiëntie van Justitie (CEPEJ). Binnen de Europese Unie hebben de gemeenschappelijke markt en het vrije verkeer van personen ook gezorgd voor een versnelling in de samenwerking op het gebied van het justitie. Daarnaast handhaven nationale rechters de geharmoniseerde materiële regels ook daadwerkelijk, voor onderwerpen die uiteenlopen van het mededingingsrecht tot consumentenbescherming. Bovendien heeft het juridisch bindende karakter van het Handvest van de Grondrechten ervoor gezorgd dat er meer aandacht is gekomen voor het opstellen van strengere normen op het gebied van justitie door heel Europa. Om deze reden monitort een aantal Europese instellingen, waaronder de Europese Commissie, het werk van nationale rechterlijke machten nu actief, en worden er analytische beoordelingen uitgevoerd.

Formeel gezien is de belangrijkste juridische norm voor het beoordelen van de prestaties van nationale civiele rechtssystemen in Europa op dit moment zonder twijfel Artikel 6 van het Europese Verdrag voor de Rechten van de Mens, over het recht op een eerlijk proces, zoals uitgelegd door het Europese Hof voor de Rechten 
van de Mens. In de afgelopen twee decennia in het bijzonder is de rechtspraak rondom dit artikel enorm uitgebreid, waardoor het inmiddels vele aspecten van het civiele proces omvat. Hierbij is het belangrijk dat het Hof, naast het vaststellen van minimumvereisten voor de eerlijkheid van een proces, ook bepaalde minimumnormen voor institutionele bevoegdheden heeft vastgesteld die in Europese landen dienen te zijn gewaarborgd. Door de opname van deze jurisprudentie in het primaire recht van de Europese Unie is het belang van deze jurisprudentie nu nog groter. Zowel de EU-instituties als lidstaten hebben hiermee binnen het raamwerk van juridische structuren, voortkomend uit EU-verdragen, namelijk verdere verplichtingen op zich genomen wat betreft het respecteren van deze criteria.

Om de doctrines van het Straatburgse Hof te evalueren, stelt deze studie een belangrijk operationeel concept voor - de effectiviteit van het civiel recht - die als leidende norm kan worden toegepast als evaluatiecriterium voor de prestaties van civielrechtelijke instituties. Rekening houdend met de verschillende evaluatierapporten over civiele rechtssystemen in verschillende landen, alsmede met juridische en economische inzichten, wordt gesteld dat een gemeenschappelijke basis voor effectiviteit kan bestaan uit drie criteria of parameters, te weten de duur, kosten, en voorspelbaarheid van processen. Deze parameters vormen niet alleen een lijst met drempels, maar brengen ook mogelijke onderlinge interacties tussen beleidskeuzes in kaart. Het effectiviteitsconcept impliceert daarmee een bijbehorend beleidsmodel met een redelijke en evenredige nadruk op de drie parameters, maar waarbij ook een balans met billijkheidseisen wordt gewaarborgd. Wanneer effectiviteit wordt geformuleerd als juridisch argument, moet een analyse van de interpretatieve praktijk, met name op het gebied van mensenrechten, rekening houden met deze drie parameters, en zorgen voor een samenhang en balans met beleidskeuzes bij het bepalen van de respectievelijke drempels.

De eerste parameter, duur, is als enige expliciet gebaseerd op Artikel 6 uit het Verdrag, en garandeert het recht op een proces binnen een "redelijke termijn". Deze vereiste is lang één van de belangrijkste werkterreinen van het Straatsburgse Hof geweest, wat niet alleen aangeeft dat vertragingen een belangrijk probleem zijn in veel Europese civiele rechtssystemen, maar ook dat pogingen om hier op Europees niveau adequate oplossingen voor te vinden, niet erg succesvol zijn geweest. Het Hof geeft landen een aanzienlijke speelruimte bij het organiseren van hun rechtssystemen, en heeft, in het bijzonder, geen uitspraken gedaan over mogelijke onderliggende oorzaken van vertragingen. Ook is het Hof terughoudend bij het ondernemen van actie tegen veelvuldige schendingen van de redelijke termijnvereiste in rechtssystemen waar vertragingen veel voorkomen. Het scheiden van uitspraken over vertragingsproblemen voor individuele rechtspartijen en bredere juridische beleidsvragen - zowel op het vlak van de processtructuur binnen het rechtssysteem als wat betreft begrotingsondersteuning - lijkt de complexiteit en 
systematische aard van de onderliggende problemen te zijn gaan overschaduwen. Ook wordt hierdoor de aandacht afgeleid van broodnodige systeemhervormingen naar meer individuele oplossingen, die echter alleen werken bij vertragingen in geïsoleerde gevallen.

De tweede parameter, kosten, heeft ook gezorgd voor veel jurisprudentie. Het Hof in Straatsburg erkende al vroeg dat het principe van een eerlijk proces in een beperkt aantal omstandigheden vereist dat economisch zwakkere rechtspartijen steun krijgen uit publieke middelen, maar dat het minder complex maken van processen tegelijkertijd ook een mogelijke oplossing kon bieden. Later breidde het Hof haar jurisprudentie geleidelijk uit tot een reeks vereisten met betrekking tot rechtsbijstand, waarbij een reeks verwante zaken de rechten van landen afbakende wat betreft eisen aan het ex ante betalen van griffierechten. Wat rechtsbijstand betreft uitte het Hof zich gaandeweg steeds specifieker, en werden er voor sommige rechtssystemen zelfs specifieke vormen en regels voorgeschreven met betrekking tot het bieden van hulp aan mensen in nood. Dit is echter niet in lijn met de eerdere, gereserveerde houding. Aan de andere kant werd het vraagstuk rondom griffierechten op een redelijk casuïstieke manier behandeld, en werden er met name schendingen vastgesteld in een aantal zaken waarin buitengewoon hoge griffierechten waren opgelegd. Hierbij werd echter geen uitgebreide algemeen toepasbare norm uiteengezet. Deze aanpak heeft er uiteindelijk voor gezorgd dat de behandeling van het kostenvraagstuk vanuit het mensenrechtenperspectief niet altijd gelijk of coherent is. Bovendien staan de beperkte en indirecte vaststellingen van schendingen in contrast met de uitgebreide en ambitieuze aanpak van vertragingen.

De derde parameter, voorspelbaarheid, werd op een andere manier behandeld. Hoewel het Hof in Straatsburg benadrukt dat jurisprudentie belangrijk is voor een zekere voorspelbaarheid van de rechtspraak, keek het niet naar belangrijke verschillen tussen Europese jurisdicties op het gebied van beroepsprocedures en jurisprudentie. Deze verschillen zijn echter cruciaal om de institutionele achtergrond van het probleem te kunnen begrijpen. In eerste instantie weigerde het Hof om een duidelijke positie in te nemen toen het werd geconfronteerd met ongelijke behandelingen van vergelijkbare situaties door nationale rechters. Daardoor werd divergentie in uitspraken, zelfs bij duidelijk ongelijke behandelingen van identieke situaties, gezien als bijwerking van hiërarchische en geregionaliseerde rechtssystemen met vele instanties en verschillende rechtelijke lagen. Pas na het beschouwen van zaken uit Oost-Europa realiseerde het Hof zich hoe dergelijke structuren - vooral in combinatie met een uiterst formalistische rechtspraktijk waarbij eerdere zaken niet worden gebruikt als richtlijn voor uitspraken - kunnen leiden tot absurde uitkomsten, en begon het nationale uitspraken als inconsistent te beoordelen. Het operationele concept dat werd gebruikt was 'rechtszekerheid', en na een aantal zaken begon het erop te lijken 
dat het Hof bereid was om voor Europese rechtssystemen een algemene vereiste op te leggen om rekening te houden met jurisprudentie. Na enkele ad-hoc pogingen besloot het Hof uiteindelijk desalniettemin om een stap terug te zetten, met een aanpak waarbij consistentie slechts een conditionele vereiste zou zijn.

De analyse van de Europese mensenrechtenpraktijk toont derhalve aan dat er nu een aantal vaststaande principes bestaat met betrekking tot de effectiviteit van het civiel recht, hoewel deze term niet expliciet wordt gebruikt. Het Hof heeft de aspecten duur, kosten, en voorspelbaarheid geïdentificeerd als belangrijk vanuit mensenrechtenperspectief, en heeft gepoogd om voor deze aspecten specifieke vereisten vast te stellen. Hierbij werd veel aandacht besteed aan de kenmerken van individuele zaken en de kenmerken van de rechtstelsels in kwestie. Desalniettemin blijkt jurisprudentie bij nader onderzoek op verschillende manieren problemen op te leveren. De leer op het gebied van de effectiviteit van rechtspraak is gefragmenteerd, en stoelt op een groot aantal ad-hoc uitspraken zonder uitgebreide systematische analyse. Dit heeft tot gevolg dat de aspecten duur, kosten, en voorspelbaarheid apart worden behandeld, in plaats van als samenhangende aspecten binnen het meer algemene vraagstuk van institutionele ontwerpen. Ook lijkt jurisprudentie op verschillende vlakken incoherent te zijn. Ten eerste heeft het Hof verschillende benaderingen gebruikt in vergelijkbare situaties en verschillende drempels toegepast voor verschillende jurisdicties. Ten tweede is er in verschillende mate aandacht besteed aan verschillende aspecten, waarbij het aspect 'duur' veel aandacht heeft gekregen, waaronder middels proefuitspraken, en zelfs een bevel van het Hof aan de lidstaten om speciale nationale rechtsmiddelen te implementeren. De aspecten 'kosten' en 'voorspelbaarheid' werden echter gezien als te afhankelijk van nationale institutionele structuren om striktere Europese standaarden op te kunnen leggen. Ten derde bestaat er ook bij het ontwikkelen van vereisten voor elk van deze drie aspecten incoherentie, omdat criteria uiteenlopen van erg algemeen en abstract, tot zeer specifiek. Aan de andere kant blijkt wat uitkomsten betreft uit de Straatsburgse jurisprudentie dat, hoewel het Hof een aantal van de meest dringende problemen op het gebied van juridische effectiviteit serieus heeft proberen aan te pakken, de inhoud van de uiteindelijk opgelegde vereisten behoorlijk beperkt is.

Er wordt aangevoerd dat het ongelijke, en voornamelijk bescheiden resultaat van deze mensenrechtenleer ten minste ten dele kan worden verklaard door de besluitvormingsaanpak, die kan worden gekarakteriseerd als overwegend formalistisch of conceptualistisch. Het Hof van Straatsburg heeft bij het analyseren van de problemen rondom langdurige, dure, en onvoorspelbare processen geen gebruik gemaakt van, of verwezen naar, de beschikbare nationale en internationale beleidsdocumenten, en heeft - enkele uitzonderingen daargelaten - ook geen gebruik gemaakt van rechtsvergelijkingen. In plaats daarvan heeft het zich bij haar beslissingen laten leiden door abstracte fantasie en abstracte concepten als 
'redelijke termijn', 'toegang tot de rechtspraak' en 'rechtszekerheid', en heeft het abstracte rechten afgeleid van het idee van een eerlijk proces door de vraag te stellen of een rechtspartij in het betreffende rechtssysteem een 'recht' heeft op bepaalde institutionele regelingen. Het Straatsburgse Hof heeft haar leer dus ontwikkeld zonder te kijken naar een referentiekader voor evaluatiecriteria of beleidskeuzes die bepalend zijn voor de institutionele context van het justitie. Meer specifiek heeft het Hof niet gekeken naar explicietere afwegingen van billijkheid en effectiviteit als leidende normen voor het opstellen van procesmodellen, en heeft het niet gekeken naar bijbehorende problemen zoals de toewijzing van middelen en prikkels, als mogelijke onderliggende problemen binnen de geanalyseerde rechtssystemen. Met andere woorden is de aanpak van het Hof voornamelijk gebaseerd op de aanname dat de aspecten vertraging, kosten, en voorspelbaarheid geformuleerd als mensenrechten - kunnen worden losgekoppeld van belangrijke beleidskeuzes over de vorming van rechtsinstellingen. In deze zin kan worden gesteld dat voor de leer die is ontwikkeld door het Straatsburgse Hof onvoldoende aandacht is besteed aan de systematische aard van problemen in de geanalyseerde rechtssystemen, en dat de gebruikte conceptualistische beslissingsaanpak kan worden bekritiseerd, omdat deze vanuit het oogpunt van politieke legitimiteit van de rechtspraak in het Europese transnationale juridische kader tot twijfelachtige resultaten leidt. 